\title{
Acute Cholecystitis Mimics Ischemic Cardiac Disease: A Case Report and Review of the Literature
}

\author{
Osman Habeeb ${ }^{1,2}$, Taj Eldeen Ebrahim ${ }^{3}$ \\ ${ }^{1}$ Faculty of Medicine and Health Sciences, University of Kassala, Kassala, Sudan \\ ${ }^{2}$ Suliman Alrajhi Medical College, Al Bukaryiha, KSA \\ ${ }^{3}$ Hepatobilliary and Liver Transplantation, Faculty of Medicine and Health Sciences, University of Kassala, \\ Kassala, Sudan \\ Email: osmanha7733@yahoo.com
}

Received 9 September 2014; revised 8 October 2014; accepted 7 November 2014

Copyright (C) 2014 by authors and Scientific Research Publishing Inc.

This work is licensed under the Creative Commons Attribution International License (CC BY).

http://creativecommons.org/licenses/by/4.0/

(c) (;) Open Access

\begin{abstract}
Chest pain and shortness of breath are typical indicators of cardiac problem and may lead to life threatening conditions. Nevertheless, these symptoms may also be associated with a non-cardiac condition clinically such as cholecystitis. We have reported 4 cases in a period of 6 months wherein the patients presented with cardiac-like symptom show ever; they were subsequently diagnosed with non-cardiac problems. Two patients presented with severe chest pain and shortness of breath mimicking angina pectoris. They were admitted to the intensive care unit for emergency management. The ECG findings were normal. There were two other patients who also presented with severe chest pain and shortness of breath with ST segment elevation during the ECG examination. The abdominal ultrasound in all the 4 patients showed typical features of acute calculus cholecystitis. All patients were young (30 - 36 years of age) females who were obese (BMI ranging from 30 34). Cholecystectomy was performed which led to improved symptoms postoperatively and ECG changes became normal.
\end{abstract}

\section{Keywords}

Cardiac, Calculus, Cholecystitis, Heart, Ischemic Cardiac Disease

\section{Introduction}

Chest pain and shortness of breath are often considered as typical indicators of cardiac problems; however, the

How to cite this paper: Habeeb, O. and Ebrahim, T.E. (2014) Acute Cholecystitis Mimics Ischemic Cardiac Disease: A Case Report and Review of the Literature. Case Reports in Clinical Medicine, 3, 609-615. 
clinical association of these symptoms with non-cardiac conditions such as cholecystitis [1] [2], pancreatitis [3] and pnemonitis [4] is also being considered as relevant. ST segment elevation is pathognomic for cardiac injury but this presentation has also been reported with other condition like cholecystitis, acute stroke, pericarditis, subarachnoid hemorrhage and hypothermia [1] [2]. It has also been associated with gastric distension [5] [6].

Acute cholecystitis is a complication of biliary tract disease characterized by a syndrome of right upper quadrant pain, fever, leukocytosis and gallbladder inflammation [7]. The patient commonly has a sudden onset of symptoms that persists for 4 hours or longer and requires clinical consultation in the emergency room. Symptoms develop from the presence of acute inflammation in the mucosa and wall of the gallbladder that causes distention of the wall and eventual ischemia. The inflammatory response ensues as sterile but can lead to secondary bacterial infection [8].

Acute cholecystitis occurs most commonly in women during the fifth and sixth decades. Approximately 120,000 cholecystectomies are performed for acute cholecystitis annually in the US. The incidence of gallstones is $0.6 \%$ in the general population and much higher in certain ethnic groups. Most patients with gallstones are asymptomatic. Of such patients, biliary colic develops in $1 \%$ to $4 \%$ annually [9].

Aim: To recognize the atypical presentation of cholecystitis in order to ensure appropriate diagnosis and treatment and prevent unnecessary therapeutic procedures.

\section{Case Report}

We report four cases during 6 months (2013-2014).

Case 1: A young (32 years) obese female (82 Kg weight, BMI 30.8) presented with sudden onset of severe chest pain that did not respond to simple analgesia with no reported exacerbating or reliving factors. The patient also complained shortness of breath, nausea and vomited twice during 24 hours before reporting for clinical examination. The patient was distressed and tachypneic, herBp was 130/70 and presentation mimicked angina pectoris. The patient was admitted to the intensive care unit for further critical management. The ECG finding was normal (Figure 1). Haematological and biochemical investigation showed neutrophelia $\left(11.6 \times 10^{9} / \mathrm{L}\right)$ with normal urea, electrolyte and liver function test. Erect chest X-ray and plain abdominal X-ray shows no abnormalities. Abdominal ultrasonogrophy revealed multiple gallstones with dilated gallbladder wall (Figure 2).

Case 2: A young (30 years) obese female (89 kg, BMI 34.2) presented with chest pain, low grade fever, shortness of breath and vomiting 4 times during 12 hours before reporting for medical care. She had a past medical history of the same presentation 5 month ago. She was admitted to the intensive care unit for further management. No chest or cardiovascular abnormalities were observed during physical examination. Her ECG was normal (Figure 3). However, her WBCs count was $\left(10.5 \times 10^{9} / \mathrm{L}\right)$ buturea, electrolyte, serum amylase and liver function test were normal with slight raise in alkaline phosphatase. Abdominal ultrasound showed distended gallbladder with a single large stone and an edematous gallbladder wall (Figure 4).

Case 3: A female (31 years) obese patient (86 kg and BMI 34) presented with severe chest pain and shortness of breath. The pain was focal and did not relive by simple analgesia. There was no coughing or abdominal pain. Her blood pressure was 130/85. Abdominal examination showed grading in the epigastric area with negative Murphye's sign. ECG showed elevation of ST segment and reported pathological Q-wave (Figure 5). Erect

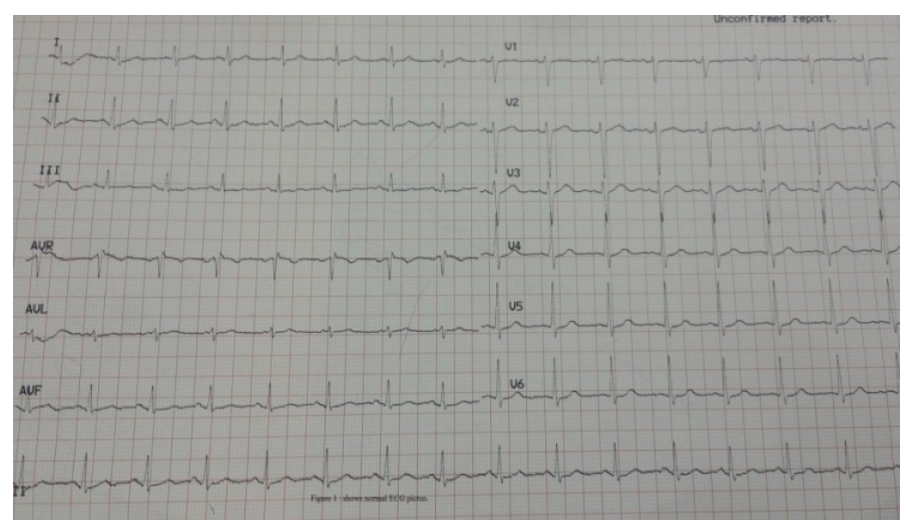

Figure 1. Shows normal ECG in case 1. 


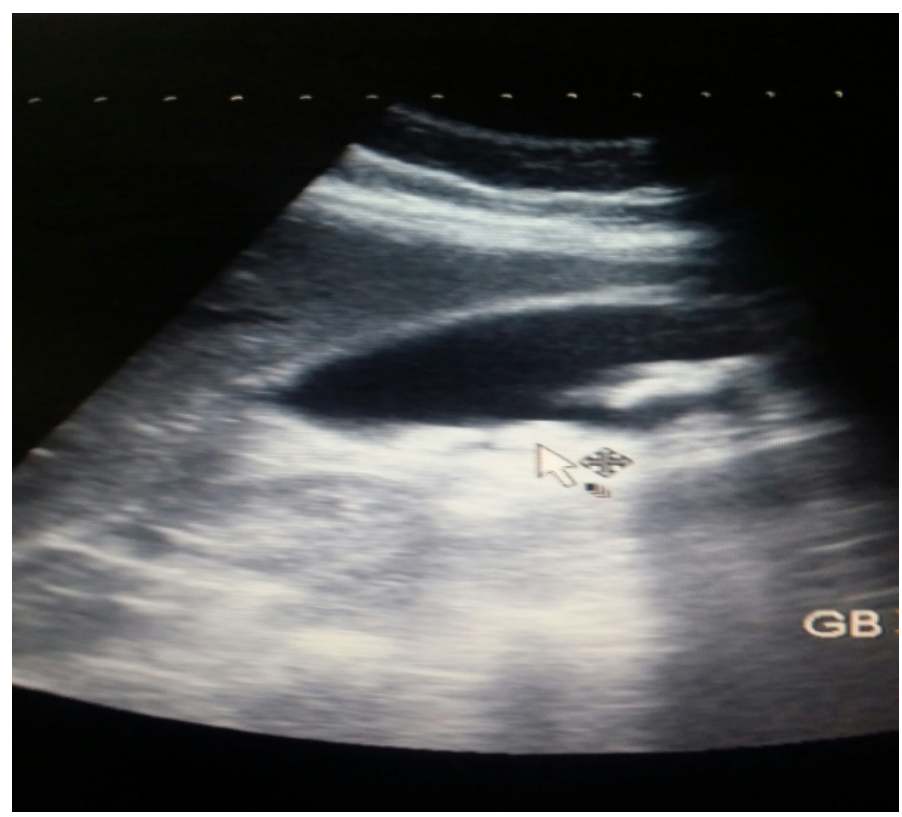

Figure 2. Shows feature of gallbladder stones.

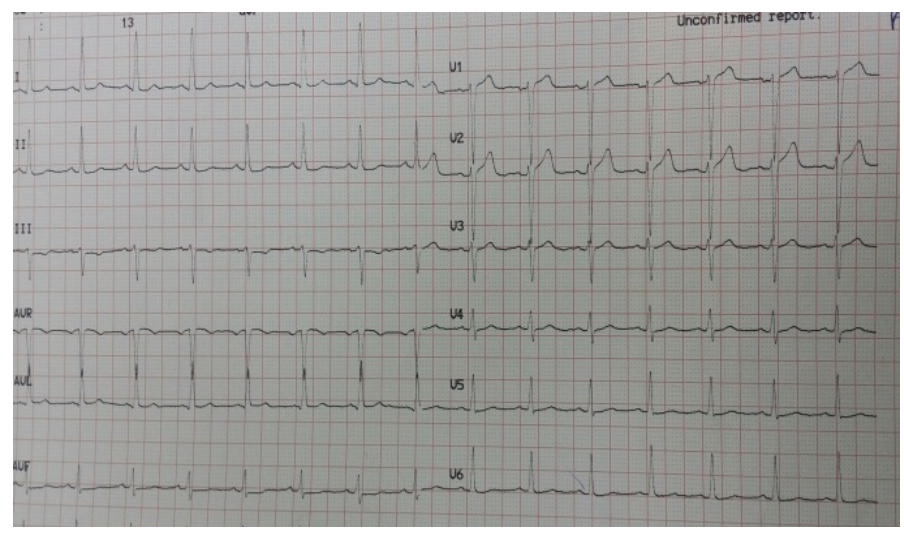

Figure 3. Shows normal ECG in case 2.

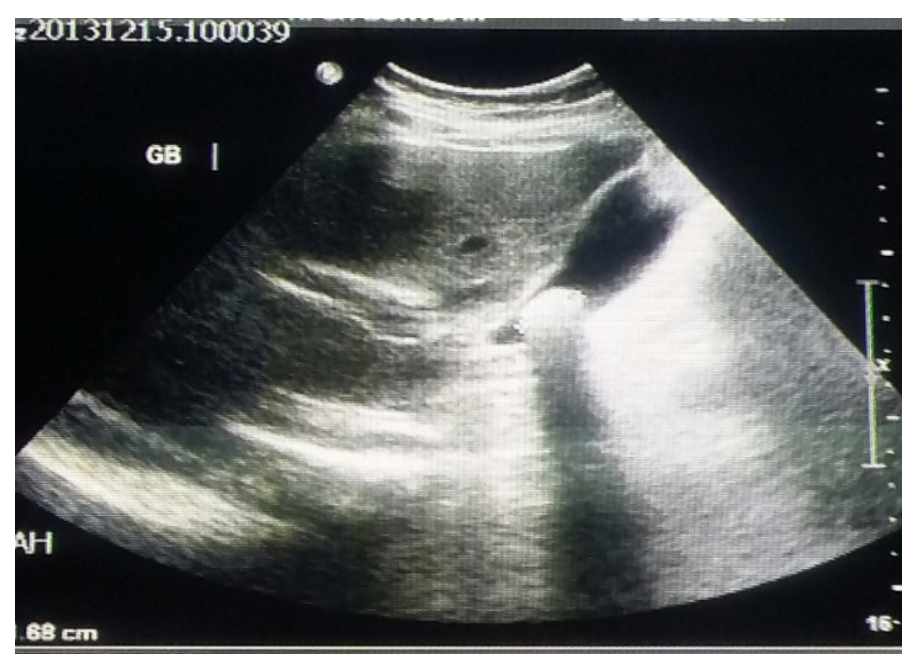

Figure 4. Showed a gallstone with acute cholecystitis. 
chest X-ray and abdominal X-ray were normal and her abdominal ultrasound showed multiple gallstones with edematous gallbladder wall.

Case 4: A female (36 years) obese patient (81 kg and BMI 33) presented with severe chest pain and shortness of breath. She had nausea and vomiting. The pain score of $8 / 10$ with no radiation. Her blood pressure was 120/ 80. Her ECG showed ST segment elevation. She had a medical history of diabetes mellitus and had a high cholesterol level. Her liver function test showed hyperbillurubinemia, but normal liver enzyme test. Abdominal ultrasound showed multiple gallstones with features of acute cholecystitis (Figure 6).

After resuscitation and management of the acute phases; all patients underwent cholecystectomy subsequent to which the symptoms of chest pain and shortness of breathing were relived completely. The ECG changes returned to normal. The patients in the first and third cases were found to have multiple large cholesterol gallstones (Figure 7 \& Figure 8), patient in the second case had a single large cholesterol gallstone (Figure 9) whereas the patient in case 4 had multiple moderate size cholesterol stones (Figure 10).

The patients were examined clinically, radiologically and ECG following surgery and during their follow-up there were no reported symptoms of chest pain, shortness of breath or ECG changes after 4 - 6 months of follow up.

\section{Discussion}

All our reported four patients were women, young ages ranges from 30 - 36 years (medium 33 years) and obese with BMI ranging between 30 - 34.2 (medium 32.1). Our patients were the youngest in the reported cases in the

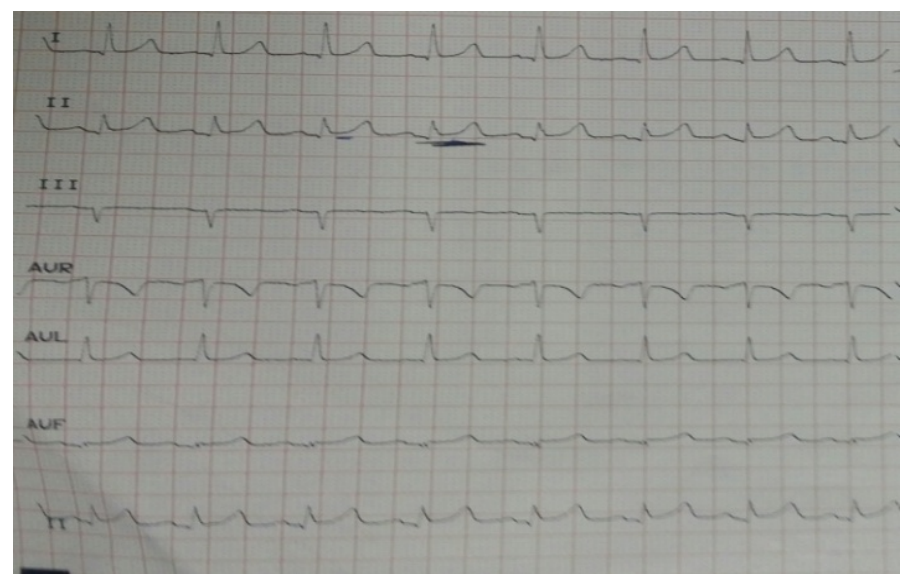

Figure 5. Showed ST segment elevation in lead II.

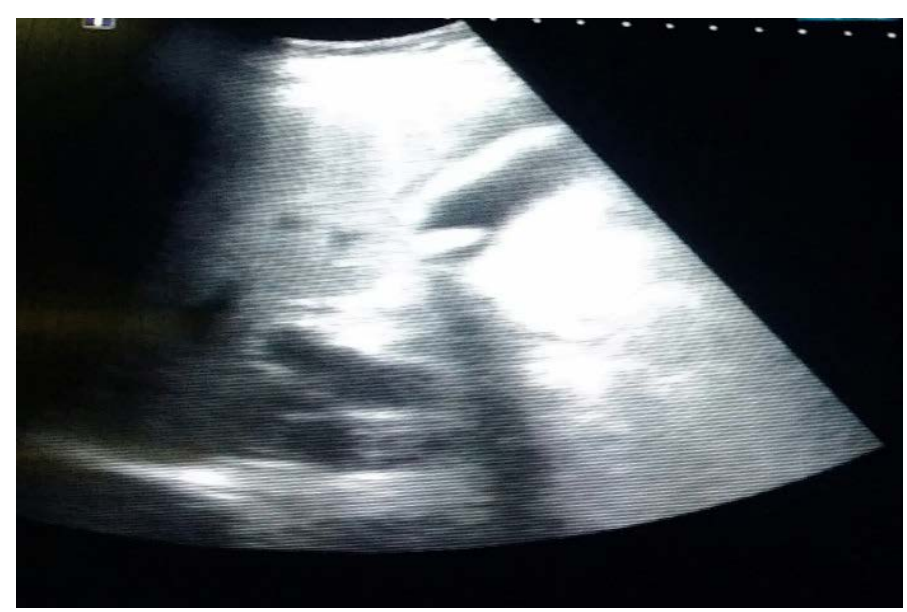

Figure 6. Ultrasound showed gallbladder stones and acute inflammation. 


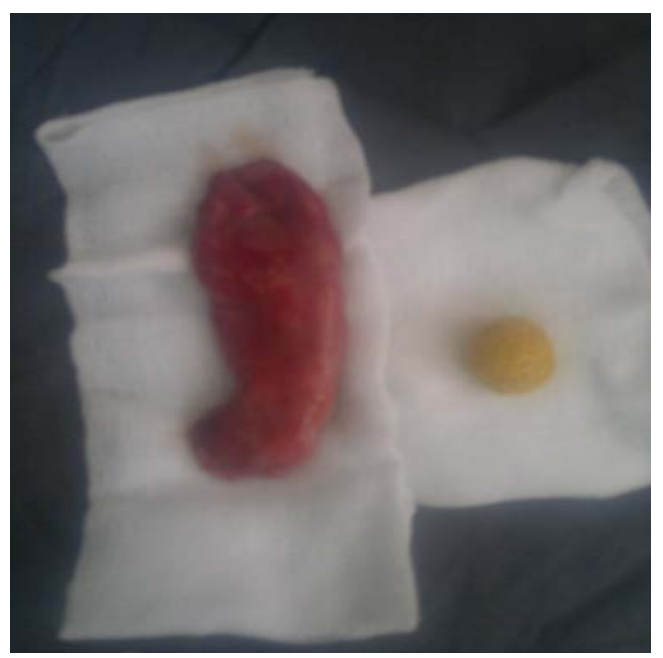

Figure 7. Resected inflamed gallbladder with multiple medium size cholesterol stones.

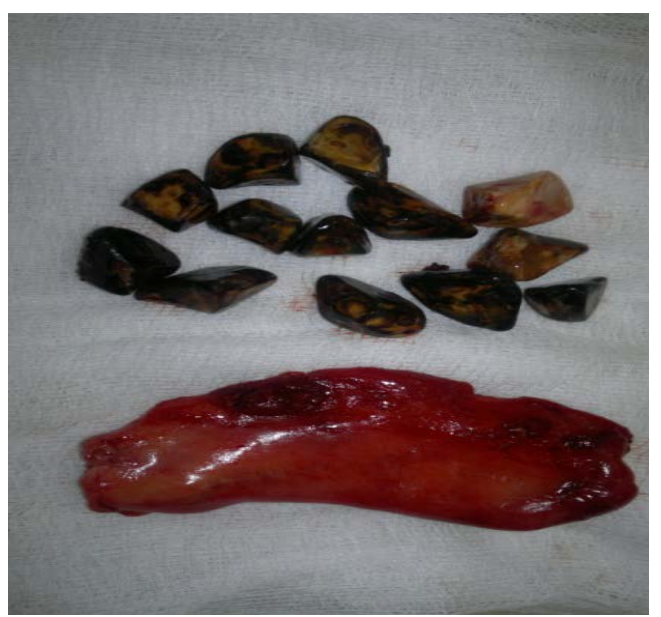

Figure 8. Resected inflamed gallbladder with solitary cholesterol stone.

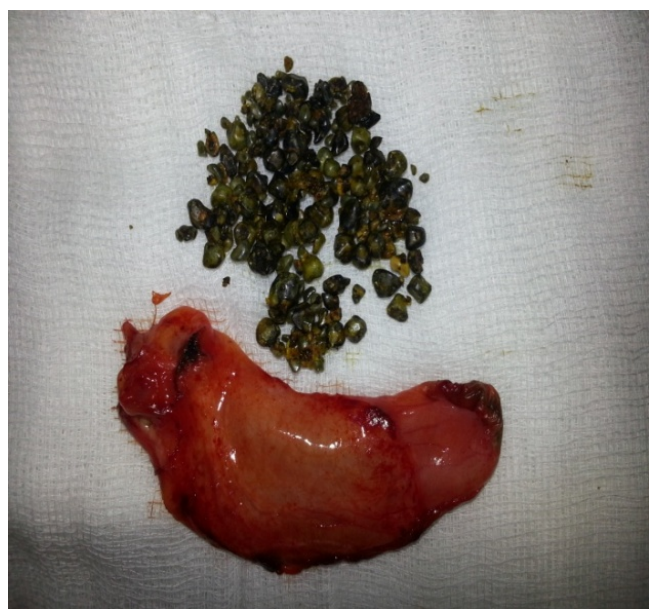

Figure 9. Resected inflamed gallbladder with multiple small size cholesterol stones. 


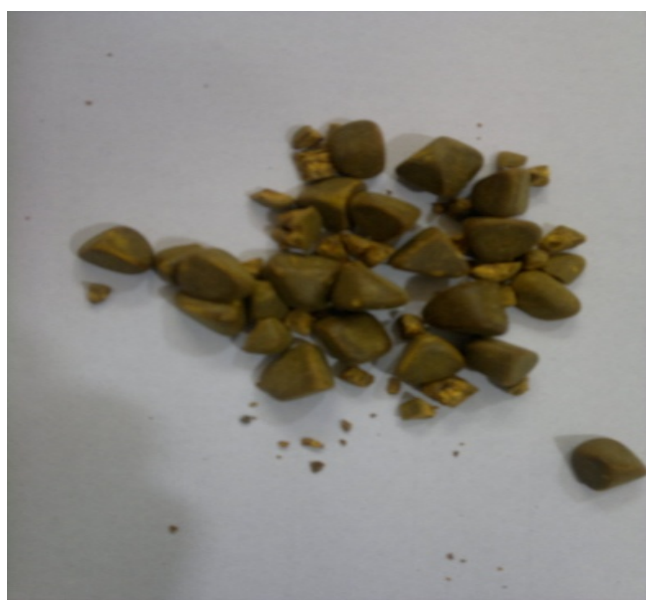

Figure 10. Multiple medium sized gallstone.

literature, all were women and all had a cholesterol type of gallstones. Two of our patients had ECG changes that resolved completely after cholecystectomy.

Six previous cases of ST segment elevation attributed to cholecystitis were reported in the literature [2] [5][10] with patients ages ranging from 46 - 64 years and five of them were male [2]-[10]. Five of the patients from these studies presented with chest pain and one with abdominal pain. Two of these patients underwent cardiac catheterization and one had received a thrombolytic therapy. In the patients with ECG changes, two patients had resolution of the ECG changes after cholecystectomy and two had resolution of ECG changes without further treatment and one patient receiving antibiotictreatment [2].

Although the ECG changes attributed to cholecystitis have been shown to be correctable with surgery and atropine treatment [2] [11] [12], the pathological mechanism underlying the ECG changes remains unknown [2]. However, the ECG changes observed can be explained by the effect on the coronary blood flow [2] [13]. Gallbladder distension had a role in tachycardia, increasing blood pressure and increase in plasma renin level [2] [10] as well as coronary artery blood flow; these finding had been demonstrated in several animal studies [2] [7] [12] [14] [15].

Demarche M.S. et al. reported a 75-year-old patient who presented with abdominal pain, abdominal tenderness and positive Murphy's sign but with associated ST-Segment elevation. The ECG changes in the patient were interpreted due to cholecystitis and gallbladder distension and gave elevation of troponin-I [7]. Increase in troponin-I level is commonly associated with myocardial cell injury [7]. Similarly, two other reports have described increase in troponin-I levels in patients with acute cholecystitis but without any ECG changes [7] [16].

\section{Conclusion}

Acute cholecystitis is a common and important acute surgical condition that necessitates a proper recognition and diagnosis. Similarity with acute cardiac diseases may lead to unnecessary management such as thrombolytic therapy with serious consequences for the patient as well as economic impaction and waste of resources.

\section{Acknowledgements}

We acknowledge Dr. Haider, H. at SRC, KSA for his contribution in revision of the manuscript.

\section{References}

[1] Ryan, E.T., Pak, P.H. and DeSancts, R.W. (1992) Myocardial Infarction Mimicked by Acute Cholecystitis. Annals of Internal Medicine, 116, 218-220. http://dx.doi.org/10.7326/0003-4819-116-3-218

[2] Nasir Javed, M., During, S.J., Sweet, J.M. and Cation Lannine, J. (2006) Chest Pain and ST Segment Elevation Attributable to Cholecystitis: A Case Report. Military Medicine, 171, 1255-1258.

[3] Pazzilli, R., Barakat, B. and Bartaccini, B. (1963) Electrocardiographic Abnormalities in Acute Pancreatitis. European Journal of Emergency Medicine, 598-602. 
[4] Edat, A.M., Feldman, C., Skoularigis, J. and Swiz, S. (1993) Astudy of Acute Community-Acquired Pneumonia, Including Details of Cardiac Changes. Quarterly Journal of Medicine, 86, 669-675. http://dx.doi.org/10.1093/qjmed/86.10.669

[5] Patel, N., Ariyarathenam, A., Davies, W. and Harris, A. (2011) Acute Cholecystitis Leading to Ischemic ECG Changes in a Patient with No Underlying Cardiac Disease. Journal of the Society of Laparoendoscopic Surgeons, 15, 105-108. http://dx.doi.org/10.4293/108680811X13022985131534

[6] Frais, M.A. and Rodgers, K. (1990) Dramatic Electrocardiographic T-Waves Changes Associated with Gastric Distension. Chest, 98, 489-490. http://dx.doi.org/10.1378/chest.98.2.489

[7] Demerchi, M.S., Regusci, L. and Fasolini, F. (2012) Electrocardiographic Changes and False-Positive Troponin I in a Patient with Acute Cholecystitis. Case Reports in Gastroenterology, 6, 410-414. http://dx.doi.org/10.1159/000339965

[8] Fred, F. (2014) Ferri, Ferri’s Clinical Advisor. Cholecystitis, 281-281.

[9] Elsey, J.K. and Schmidt, D.R. (2014) Cholecystitis. Current Surgical Treatment, 387-391

[10] Cohen, O.J. (1991) Electrocardiographic ST Segment Elevation in Cholecystitis. Hospital Physician, 27, 15-18.

[11] Kaufman, J.M. and Lubera, R. (1967) Preoperative Use of Atropine and Electrocardiographic Changes. Journal of the American Medical Association, 200, 109-112. http://dx.doi.org/10.1001/jama.1967.03120160063006

[12] Brettwlesser, E.R. (1947) Electrocardiographic Observation in Chronic Cholecystitis before and after Surgery. The American Journal of the Medical Sciences, 213, 598-602. http://dx.doi.org/10.1097/00000441-194705000-00011

[13] Gilbert, N.C. (1942) Influence of Extrinsic Factors on the Coronary Flow and Clinical Course of Heart Disease. Bulletin of the New York Academy of Medicine, 18, 83-92.

[14] Vacca, G., Battaglia, A. and Mary, D.A. (1994) Tachycardia and Pressure Response to Distension of the Gallbladder in the Anaesthetized Pig. Medical Science Research, 22, 697-699.

[15] Ordway, G.A. and Longhurst, J.C. (1983) Cardiovascular Reflexes Arising from the Gallbladder of the Cat. Effects of Capsaicin, Bradykinin, and Distension. Circulation Research, 52, 26-35. http://dx.doi.org/10.1161/01.RES.52.1.26

[16] Banerjee, S., Linder, M.W. and Singer, I. (2001) False-Positive Troponin I in a Patient with Acute Cholecystitis and Positive Rheumatoid Factor Assay. Cardiology, 95, 170-171. http://dx.doi.org/10.1159/000047367 
Scientific Research Publishing (SCIRP) is one of the largest Open Access journal publishers. It is currently publishing more than 200 open access, online, peer-reviewed journals covering a wide range of academic disciplines. SCIRP serves the worldwide academic communities and contributes to the progress and application of science with its publication.

Other selected journals from SCIRP are listed as below. Submit your manuscript to us via either submit@scirp.org or Online Submission Portal.
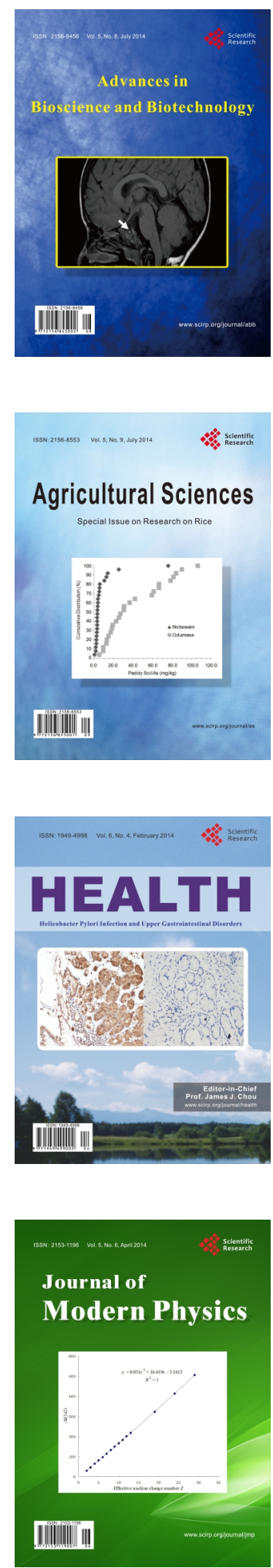
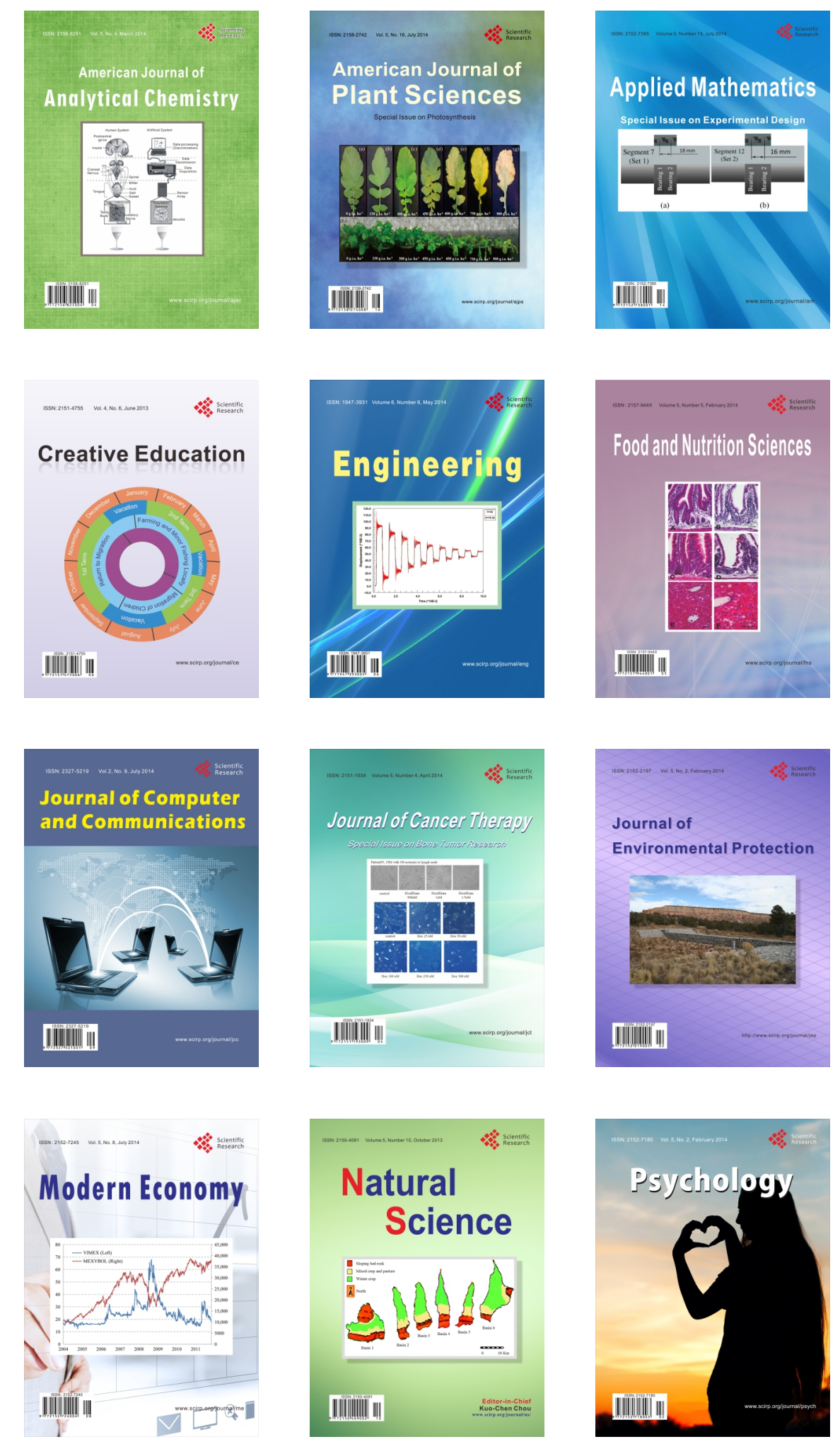